\title{
NLO QCD Corrections to Electroweak Higgs Boson Plus Three Jet Production at the LHC
}

\section{Francisco Campanario}

Theory Division, IFIC, University of Valencia-CSIC

E-46100 Paterna, Valencia, Spain

E-mail: francisco.campanariodific.uv.es

\section{Terrance M. Figy*}

School of Physics and Astronomy, The University of Manchester

Manchester, M13 9PL, United Kingdom

E-mail: Terrance.Figy@hep.manchester.ac.uk

\section{Simon Plätzer}

Theory Group, DESY

D-22607 Hamburg, Germany

E-mail:simon.plaetzer@desy.de

\section{Malin Sjödahl}

Department of Astronomy and Theoretical Physics, Lund University

SE-22362 Lund, Sweden

malin.sjodahl@thep.lu.se

The implementation of the full next-to-leading order (NLO) QCD corrections to electroweak Higgs boson plus three jet production at hadron colliders such as the LHC within the Matchbox NLO framework of the Herwig ++ event generator is discussed. We present numerical results for integrated cross sections and kinematic distributions.

11th International Symposium on Radiative Corrections (Applications of Quantum Field Theory to Phenomenology) (RADCOR 2013),

22-27 September 2013

Lumley Castle Hotel, Durham, UK

\footnotetext{
*Speaker.
} 


\section{Introduction}

In recent reports, the ATLAS and CMS Collaborations have confirmed with greater confidence the existence of a new boson with a mass in the range of $125-126 \mathrm{GeV}$ and a spin different from one $[1,2]$, and suggest that the new particle exhibits production and decays similar to a Standard Model (SM) Higgs boson [3, 4, 5, 6]. Further, reports from the ATLAS and CMS Collaborations indicate that current data provide evidence for a spin-0 Higgs boson with positive parity $[7,8]$ and have performed measurements of Higgs boson production and couplings for di-boson final states $[9,10]$.

The production of a Higgs boson via vector boson fusion (VBF), i.e. the $t$-channel $\mathscr{O}\left(\alpha_{Q E D}^{3}\right)$ reaction $q q \rightarrow q q H$, is an essential channel at the LHC for constraining Higgs boson couplings to gauge bosons and fermions. With the current experimental data from the LHC, the ATLAS Collaboration find $3 \sigma$ evidence [9] for Higgs boson production via VBF while the CMS Collaboration find $1.3 \sigma$ evidence [11]. The observation of two forward tagging jets in Higgs boson production via VBF is crucial for the reduction of backgrounds. The additional requirement that there is no extra radiation within the rapidity gap between the forward tagging jets known as the central jet veto (CJV) proposal leads to a further suppression of QCD backgrounds [12, 13, 14]. Further, the CJV proposal has been shown to be effective in reducing contamination from gluon fusion production of Higgs boson in association of two jets (GF $H j j)$ [15, 16, 17, 18, 19].

In order to exploit the CJV strategy for Higgs boson coupling measurements, the reduction factor due the CJV must be accurately known. The fraction of VBF Higgs boson events with an additional jet in the rapidity gap region, i.e, the ratio the Higgs boson plus three jet (EW $H j j j$ ) production cross section to the inclusive Higgs boson plus two jet (EW $H$ $j$ j) cross section, between the two tagging jets provides the relevant information. Recently, GF $H j j j$ production has been computed within the heavy top effective theory approximation to next-to-leading order (NLO) in perturbative QCD [20]. The usage of the heavy top effective theory approximation for $H j j(j)$ has been validated against $H j j(j)$ amplitudes where the top mass dependences has been kept in Refs. [21, 22].

The NLO QCD corrections for $H j j j$ via VBF were presented in Ref. [23, 24] within the $t$-channel approximation and without the inclusion of pentagon and hexagon one-loop Feynman diagram topologies (Figure 1, last two diagrams) and the corresponding real emission contributions, which were estimated to be at the per mille level. Given the relevance to the determination of Higgs boson couplings, we will present results from Ref. [25], where the full NLO QCD corrections to the $\mathscr{O}\left(\alpha_{s} \alpha_{E W}^{3}\right)$ production of a Higgs boson in association of three jets for the first time had been performed.

This proceedings is organized as follows: Section 2 provides details of our NLO calculation, Section 3 presents numerical results, and in Section 4 we conclude.

\section{Calculational Details}

For the computation of the leading order (LO) $2 \rightarrow H+n(n=2,3,4)$ parton matrix elements, we utilized the built-in spinor helicity library of the Matchbox module of the Herwig++ event generator [26] in order to construct the full amplitude from hadronic currents [27]. For the 
computation of the interference of the virtual one-loop amplitude with the Born amplitude, we employed the helicity amplitude technique described in Ref. [28]. This resulted in two independent versions of the Born amplitudes which provided a valuable internal consistency check of our implementation. The LO $2 \rightarrow H+n(n=2,3,4)$ parton matrix elements were cross checked against Sherpa [29, 30] and Hawk [31, 32]. Catani-Seymour dipole subtraction terms [33] have been generated automatically by the Matchbox module [27]. In order to generate phase points more efficiently, we utilized a diagram-based multichannel phase space sampler [27]. We have used inhouse routines for the one-loop virtuals, extending the techniques developed in Ref. [34], in order perform the reduction of the tensor integrals down to a basis of scalar one-loop integrals. The resulting amplitudes have been cross checked against GoSam [35]. A representative set of one-loop Feynman diagram topologies that contribute to the virtual corrections are depicted in Figure 1.
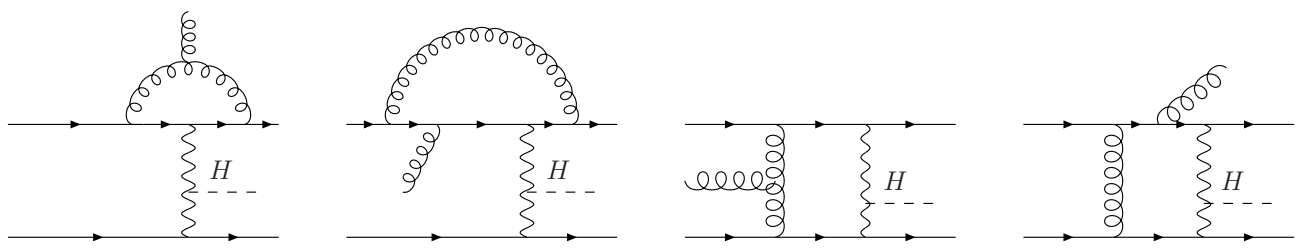

Figure 1: A subset of one-loop Feynman diagram topologies for EW $H j j j$ production.

We have employed the complex mass scheme as described in Ref. [36, 37] in order to include finite width effects in gauge boson propagators. We use the program OneLOop [38] in order to compute one-loop scalar loop integrals with complex masses. We use the Passarino-Veltman approach [39] to reduce tensor coefficients up to four-point functions, and use the Denner-Dittmaier scheme [40], following the layout and notation of [34] to numericaly evaluate the five and six point coefficients.

In order in ensure the numerical stability of our code, a test based on Ward identities has been implemented [34]. These Ward identities are checked for each phase space point and Feynman diagram, at the expense of a small increase in computing time. If the Ward identity test fails, the amplitudes of the gauge related topology are set to zero. The occurrence in which the Ward indenties are violated is at the per-mille level, hence, under control. The tensorial reduction method employed here has, also, been successfully applied in other scattering processes with $2 \rightarrow 4$ kinematics [41, 42]. In the work presented here, the method is applied for the first time to a process which involves loop propagators with complex masses.

The color algebra associated with the computation of color correlated Born matrix elements has been performed using Colorfull [43] and cross checked using ColorMath [44]. As a further check on the framework, we have implemented the corresponding calculation of electroweak $H j j$ production and, subsequently, performed cross checks against Hawk [31, 32] and VBFNLO [45]. We have designated the implementation of the NLO corrections in perturbative QCD for electroweak Higgs boson plus two and three jet production in the Mat chbox framework as HJets++. 


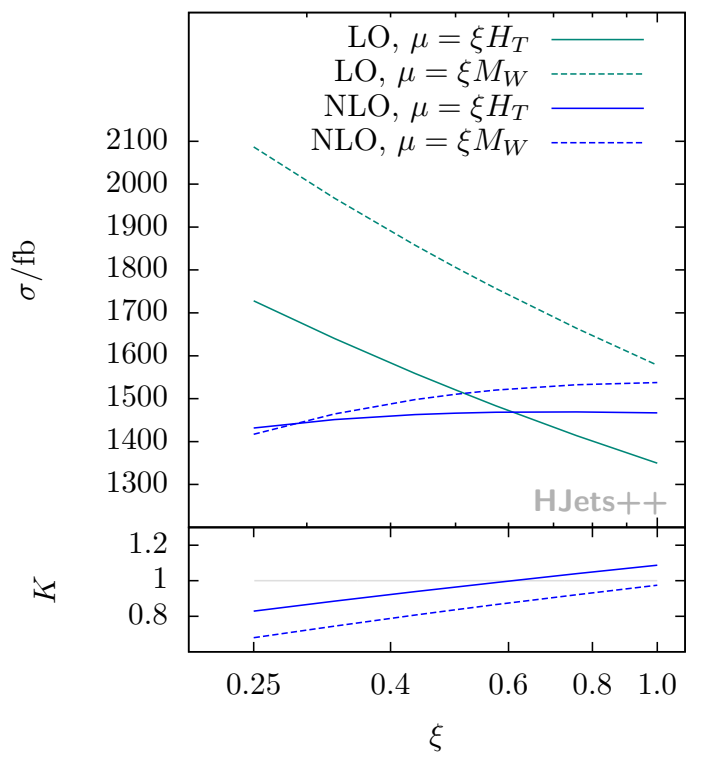

Figure 2: The $H j j j$ inclusive total cross section (in fb) at $\mathrm{LO}$ (cyan) and at NLO (blue) for the scale choices, $\mu=\xi M_{W}$ (dashed) and $\mu=\xi H_{T}$ (solid). Also, shown is the $K$-factor, $K=\sigma_{N L O} / \sigma_{L O}$ for $\mu=\xi M_{W}$ (dashed) and $\mu=\xi H_{T}$ (solid).

\section{Results}

The results presented here are computed for a LHC of center-of-mass energy $\sqrt{s}=14 \mathrm{TeV}$. We use Herwig $++[26]$ to generate and analyze NLO events. We do not include parton shower and hadronization effects in our simulations. Hard final-state partons are recombined into jets according to the anti- $k_{T}$ algorithm [46] using Fast Jet [47] with $D=0.4, E$-scheme recombination. We select events with at least three jets with transverse momentum $p_{T, j} \geq 20 \mathrm{GeV}$ and rapidity $\left|y_{j}\right| \leq$ 4.5. Jets are ordered from highest to lowest in $p_{T}$.

We use the CT10 [48] parton distribution functions with $\alpha_{s}\left(M_{Z}\right)=0.118$ at NLO, and CTEQ6L1 set [49] with $\alpha_{s}\left(M_{Z}\right)=0.130$ at LO. We use the five-flavor scheme. We choose $m_{Z}=91.188 \mathrm{GeV}$, $m_{W}=80.419002 \mathrm{GeV}, m_{H}=125 \mathrm{GeV}$ and $G_{F}=1.16637 \times 10^{-5} \mathrm{GeV}^{-2}$ as electroweak input parameters and derive the weak mixing angle $\sin \theta_{W}$ and $\alpha_{Q E D}$ from SM tree level relations. All fermion masses (except the top quark) are set to zero and the CKM matrix is taken to be diagonal. Widths are fixed to the following values: $\Gamma_{W}=2.0476 \mathrm{GeV}$ and $\Gamma_{Z}=2.4414 \mathrm{GeV}$.

In Figure 2, we show the LO and NLO total cross-sections for inclusive cuts for different values of the factorization and renormalization scale varied around the central scale, $\mu$ for two scale choices, $M_{W} / 2$, and the scalar sum of the jet transverse momenta, $\mu_{R}=\mu_{F}=\mu=H_{T} / 2$ with $H_{T}=\sum_{j} p_{T, j}$. In general, we see a somewhat increased cross section and - as expected decreased scale dependence in the NLO results. We also note that the central values for the various scale choices are closer to each other at NLO. The uncertainties obtained by varying the central value a factor two up and down are around 30\% (24\%) at LO and 2\% (9\%) at NLO using $H_{T} / 2$ $\left(M_{W} / 2\right)$ as scale choice. For the scale choice $\mu=H_{T} / 2$, we obtained $\sigma_{L O}=1520(8)_{-171}^{+208} \mathrm{fb}$ and $\sigma_{N L O}=1466(17)_{-35}^{+1} \mathrm{fb}$. Studying differential distributions, we find that these generally vary less using the scalar transverse momentum sum choice, used from now on. 

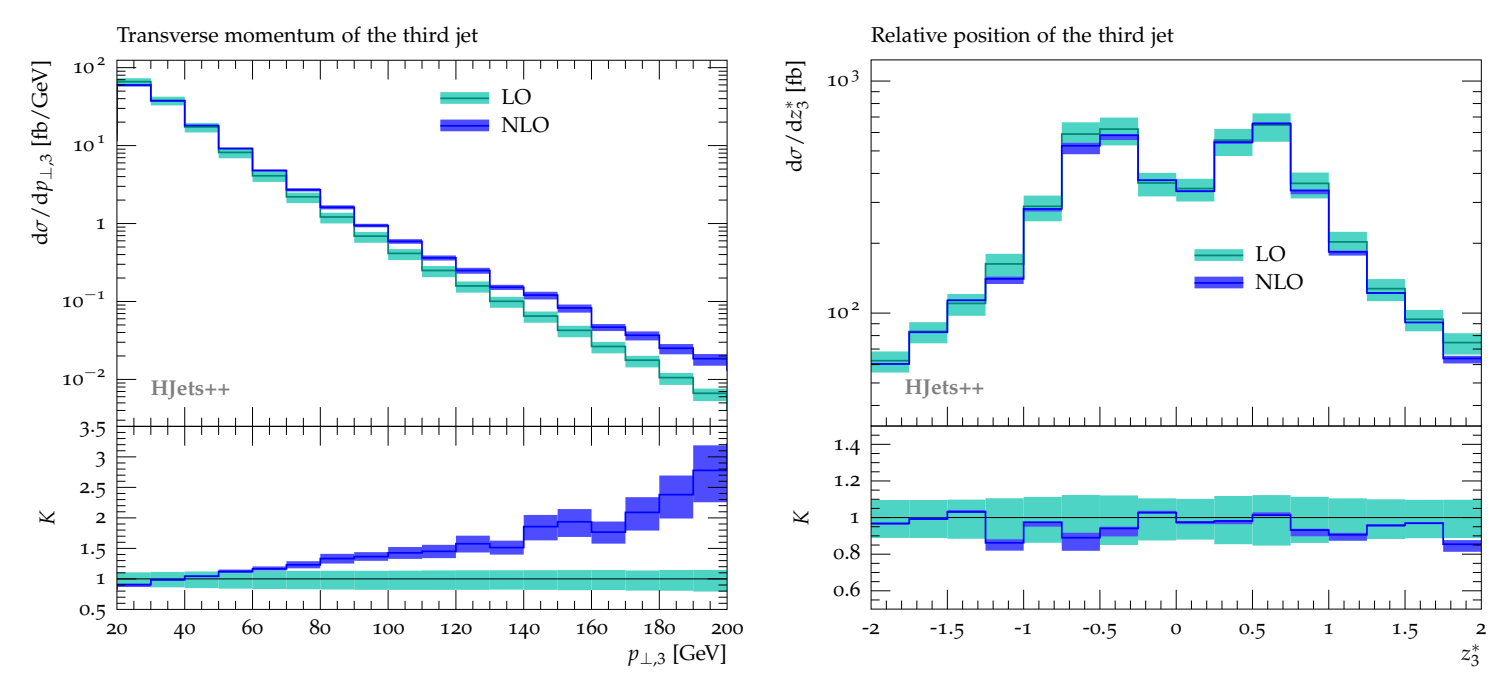

Figure 3: Differential cross section and $K$ factor for the $p_{T}$ of the third hardest jet (left) and the normalized centralized rapidity distribution of the third jet w.r.t. the tagging jets (right). Cuts are described in the text. The bands correspond to varying $\mu_{F}=\mu_{R}$ by factors $1 / 2$ and 2 around the central value $H_{T} / 2$.
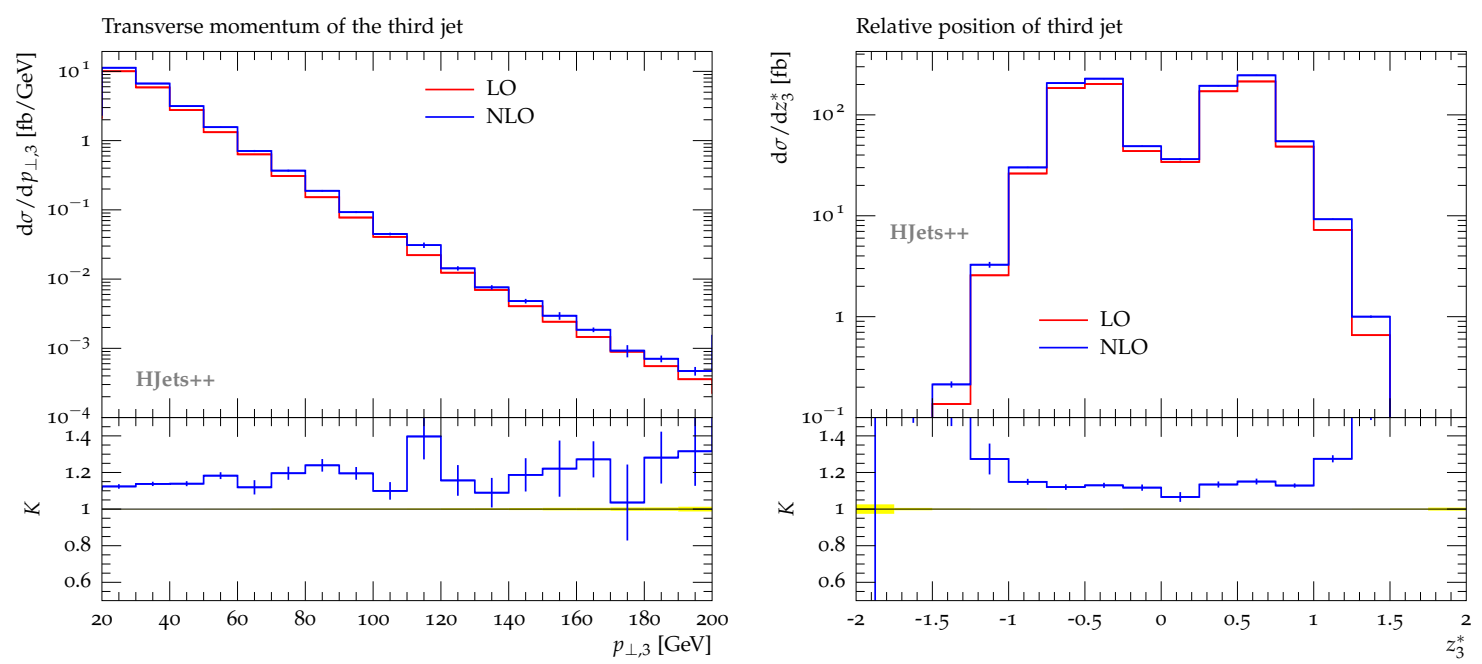

Figure 4: Differential cross section and $K$ factor for the $p_{T}$ of the third hardest jet (left) and the normalized centralized rapidity distribution of the third jet w.r.t. the tagging jets (right) with $\mu_{R}=\mu_{F}=H_{T}$. Beyond the inclusive cuts described in the text, we include the set of VBF cuts: $m_{12}=\sqrt{\left(p_{1}+p_{2}\right)^{2}}>600 \mathrm{GeV}$ and $\left|\Delta y_{12}\right|=\left|y_{1}-y_{2}\right|>4.0$.

On the left-hand side of Figure 3, the differential distribution of the third jet, the vetoed jet for a CJV analysis, is shown. Here we find large $K$ factors in the high energy tail of the transverse momentum distribution. However, when VBF cuts ${ }^{1}$ are included the $K$ factor is almost flat for the transverse momentum of the third jet (see the left-hand side of Figure 4). On the right-hand side of Figure 3, we show the normalized centralized rapidity distribution of the third jet w.r.t. the tagging jets, $z_{3}^{*}=\left(y_{3}-\frac{1}{2}\left(y_{1}+y_{2}\right)\right) /\left(y_{1}-y_{2}\right)$. This variable beautifully displays the VBF nature

\footnotetext{
${ }^{1}$ For the VBF cuts we have chosen to include the following cuts in addition to the inclusive cuts described in the main text : $m_{12}=\sqrt{\left(p_{1}+p_{2}\right)^{2}}>600 \mathrm{GeV}$ and $\left|\Delta y_{12}\right|=\left|y_{1}-y_{2}\right|>4.0$
} 
present in the process. One clearly sees how the third jet tends to accompany one of the leading jets appearing at $1 / 2$ and $-1 / 2$ respectively. This effect is more pronounced when VBF cuts are applied (see Figure 4), and should be contrasted with the gluon fusion production mechanism where QCD radiation in the rapidity gap region between the leading two jets will be much more common due to the $t$-channel color flow of the process $[16,18,21,20]$.

\section{Conclusions}

In this proceedings, complete results at NLO QCD for electroweak Higgs boson production in association with three jets have been discussed. The NLO corrections to the total inclusive cross section are moderate for inclusive cuts and the scale choice of $H_{T} / 2$. However, for the scale choice of $M_{W} / 2$, the NLO corrections can be more significant. The scale uncertainty decreases from around $30 \%(24 \%)$ at LO down to about $2 \%(9 \%)$ at NLO using the scale choice of $H_{T} / 2$ $\left(M_{W} / 2\right)$. We have, also, presented numerical results showing the impact of VBF selection cuts on the transverse momentum of the third jet, $p_{T, 3}$, and its relative position w.r.t to the two leading jets, $z_{3}^{*}$ at NLO in perturbative QCD.

\section{Acknowledgments}

We are grateful to Ken Arnold for contributions at an early stage of this project and to Mike Seymour and Jeff Forshaw for valuable discussions on the subject. F.C. is funded by a Marie Curie fellowship (PIEF-GA-2011- 298960) and partially by MINECO (FPA2011-23596) and by LHCPhenonet (PITN-GA-2010-264564). T.F. would like to thank the North American Foundation for The University of Manchester and George Rigg for their financial support. S.P. has been supported in part by the Helmholtz Alliance "Physics at the Terascale" and M.S. was supported by the Swedish Research Council, contract number 621-2010-3326.

\section{References}

[1] ATLAS Collaboration Collaboration, G. Aad et al., Observation of a new particle in the search for the Standard Model Higgs boson with the ATLAS detector at the LHC, Phys.Lett. B716 (2012) 1-29, [arXiv:1207.7214].

[2] S. Chatrchyan et al., Observation of a new boson at a mass of $125 \mathrm{GeV}$ with the CMS experiment at the LHC, Phys. Lett. B 716 (Jul, 2012) 30-61. 59 p.

[3] P. W. Higgs, Broken Symmetries and the Masses of Gauge Bosons, Phys.Rev.Lett. 13 (1964) 508-509.

[4] P. W. Higgs, Broken symmetries, massless particles and gauge fields, Phys.Lett. 12 (1964) 132-133.

[5] F. Englert and R. Brout, Broken Symmetry and the Mass of Gauge Vector Mesons, Phys.Rev.Lett. 13 (1964) 321-323.

[6] G. Guralnik, C. Hagen, and T. Kibble, Global Conservation Laws and Massless Particles, Phys.Rev.Lett. 13 (1964) 585-587.

[7] ATLAS Collaboration Collaboration, G. Aad et al., Evidence for the spin-O nature of the Higgs boson using ATLAS data, arXiv: 1307.1432.

[8] Combination of standard model Higgs boson searches and measurements of the properties of the new boson with a mass near $125 \mathrm{GeV}$, Tech. Rep. CMS-PAS-HIG-13-005, CERN, Geneva, 2013.

[9] ATLAS Collaboration Collaboration, G. Aad et al., Measurements of Higgs boson production and couplings in diboson final states with the ATLAS detector at the LHC, arXiv:1307.1427. 
[10] Properties of the observed higgs-like resonance using the diphoton channel, Tech. Rep. CMS-PAS-HIG-13-016, CERN, Geneva, 2013.

[11] Update of the search for the Standard Model Higgs boson decaying into WW in the vector boson fusion production channel, Tech. Rep. CMS-PAS-HIG-13-022, CERN, Geneva, 2013.

[12] V. D. Barger, R. Phillips, and D. Zeppenfeld, Mini - jet veto: A Tool for the heavy Higgs search at the LHC, Phys.Lett. B346 (1995) 106-114, [hep-ph/9412276].

[13] D. L. Rainwater, D. Zeppenfeld, and K. Hagiwara, Searching for $H \rightarrow \tau^{+} \tau^{-}$in weak boson fusion at the CERN LHC, Phys.Rev. D59 (1998) 014037, [hep-ph / 9808468 ].

[14] D. L. Rainwater and D. Zeppenfeld, Observing $H \rightarrow W^{*} W^{*} \rightarrow e^{ \pm} \mu \mp p_{T}$ in weak boson fusion with dual forward jet tagging at the CERN LHC, Phys.Rev. D60 (1999) 113004, [hep-ph/ 9906218$].$

[15] V. Del Duca, A. Frizzo, and F. Maltoni, Higgs boson production in association with three jets, JHEP 0405 (2004) 064, [hep-ph / 0404013 ].

[16] J. R. Forshaw and M. Sjodahl, Soft gluons in Higgs plus two jet production, JHEP 0709 (2007) 119, [arXiv:0705.1504].

[17] J. R. Andersen, V. Del Duca, and C. D. White, Higgs Boson Production in Association with Multiple Hard Jets, JHEP 0902 (2009) 015, [arXiv: 0808 . 3696].

[18] B. E. Cox, J. R. Forshaw, and A. D. Pilkington, Extracting Higgs boson couplings using a jet veto, Phys.Lett. B696 (2011) 87-91, [arXiv: 1006.0986 ].

[19] S. Gangal and F. J. Tackmann, NLO Uncertainties in Higgs +2 jets from Gluon Fusion, Phys.Rev. D87 (2013) 093008, [arXiv: 1302 .5437].

[20] G. Cullen, H. van Deurzen, N. Greiner, G. Luisoni, P. Mastrolia, et al., NLO QCD corrections to Higgs boson production plus three jets in gluon fusion, Phys.Rev.Lett. 111 (2013) 131801, [arXiv:1307.4737].

[21] F. Campanario and M. Kubocz, Higgs boson production in association with three jets via gluon fusion at the LHC: Gluonic contributions, Phys.Rev. D88 (2013) 054021, [arXiv: 1306.1830 ].

[22] V. Del Duca, W. Kilgore, C. Oleari, C. Schmidt, and D. Zeppenfeld, Higgs +2 jets via gluon fusion, Phys.Rev.Lett. 87 (2001) 122001, [hep-ph/ 0105129 ].

[23] T. Figy, V. Hankele, and D. Zeppenfeld, Next-to-leading order QCD corrections to Higgs plus three jet production in vector-boson fusion, JHEP 0802 (2008) 076, [arXiv: 0710.5621$].$

[24] T. M. Figy, NLO QCD corrections to the jet activity in Higgs boson production via vector-boson fusion, Ph.D. Thesis (2006).

[25] F. Campanario, T. M. Figy, S. Plätzer, and M. Sjödahl, Electroweak Higgs Boson Plus Three Jet Production at Next-to-Leading-Order QCD, Phys. Rev. Lett. 111 (Nov, 2013) 211802, [arXiv: 1308.2932].

[26] M. Bähr et al., Herwig++ Physics and Manual, Eur. Phys. J. C58 (2008) 639-707, [arXiv:0803.0883].

[27] S. Platzer and S. Gieseke, Dipole Showers and Automated NLO Matching in Herwig++, Eur.Phys.J. C72 (2012) 2187, [arXiv:1109.6256].

[28] K. Hagiwara and D. Zeppenfeld, Amplitudes for Multiparton Processes Involving a Current at e+e-, e+- p, and Hadron Colliders, Nucl.Phys. B313 (1989) 560. 
[29] T. Gleisberg et al., SHERPA 1.alpha, a proof-of-concept version, JHEP 02 (2004) 056, [hep-ph/0311263].

[30] T. Gleisberg et al., Event generation with SHERPA 1.1, JHEP 02 (2009) 007, [arXiv: 0811.4622 ].

[31] M. Ciccolini, A. Denner, and S. Dittmaier, Strong and electroweak corrections to the production of Higgs + 2jets via weak interactions at the LHC, Phys.Rev.Lett. 99 (2007) 161803, [arXiv:0707.0381].

[32] M. Ciccolini, A. Denner, and S. Dittmaier, Electroweak and QCD corrections to Higgs production via vector-boson fusion at the LHC, Phys.Rev. D77 (2008) 013002, [arXiv: 0710.4749 ].

[33] S. Catani and M.H. Seymour, A general algorithm for calculating jet cross sections in NLO QCD, Nucl. Phys. B485 (1997) 291-419, [hep-ph / 9605323$].$

[34] F. Campanario, Towards $p p \rightarrow V V j j$ at NLO QCD: Bosonic contributions to triple vector boson production plus jet, JHEP 1110 (2011) 070, [arXiv: 1105.0920 ].

[35] G. Cullen, N. Greiner, G. Heinrich, G. Luisoni, P. Mastrolia, et al., Automated One-Loop Calculations with GoSam, Eur.Phys.J. C72 (2012) 1889, [arXiv:1111.2034].

[36] A. Denner and S. Dittmaier, The Complex-mass scheme for perturbative calculations with unstable particles, Nucl.Phys.Proc.Suppl. 160 (2006) 22-26, [hep-ph / 0605312$].$

[37] M. Nowakowski and A. Pilaftsis, On gauge invariance of Breit-Wigner propagators, Z.Phys. C60 (1993) 121-126, [hep-ph/9305321].

[38] A. van Hameren, OneLOop: For the evaluation of one-loop scalar functions, Comput.Phys.Commun. 182 (2011) 2427-2438, [arXiv:1007.4716].

[39] G. Passarino and M. Veltman, One Loop Corrections for e+ $e$-Annihilation Into mu+ mu- in the Weinberg Model, Nucl.Phys. B160 (1979) 151.

[40] A. Denner and S. Dittmaier, Reduction schemes for one-loop tensor integrals, Nucl.Phys. B734 (2006) 62-115, [hep-ph/0509141].

[41] F. Campanario, C. Englert, M. Rauch, and D. Zeppenfeld, Precise predictions for $W \gamma \gamma+$ jet production at hadron colliders, Phys.Lett. B704 (2011) 515-519.

[42] F. Campanario, M. Kerner, L. D. Ninh, and D. Zeppenfeld, WZ production in association with two jets at NLO in QCD, Phys. Rev. Lett. 111, 052003 (2013) [arXiv: 1305.1623].

[43] M. Sjodahl, ColorFull - A C++ package for color space calculations, http://colorfull.hepforge.org/.

[44] M. Sjodahl, ColorMath - A package for color summed calculations in SU(Nc), Eur.Phys.J. C73 (2013) 2310, [arXiv:1211.2099].

[45] K. Arnold, J. Bellm, G. Bozzi, F. Campanario, C. Englert, et al., Release Note - Vbfnlo-2.6.0, arXiv:1207.4975.

[46] M. Cacciari, G. P. Salam, and G. Soyez, The Anti-k(t) jet clustering algorithm, JHEP 0804 (2008) 063, [arXiv: 0802.1189$]$.

[47] M. Cacciari, G. P. Salam, and G. Soyez, FastJet User Manual, Eur.Phys.J. C72 (2012) 1896, [arXiv:1111.6097].

[48] H.-L. Lai, M. Guzzi, J. Huston, Z. Li, P. M. Nadolsky, et al., New parton distributions for collider physics, Phys.Rev. D82 (2010) 074024, [arXiv: 1007.2241$].$

[49] J. Pumplin, D. Stump, J. Huston, H. Lai, P. M. Nadolsky, et al., New generation of parton distributions with uncertainties from global QCD analysis, JHEP 0207 (2002) 012, [hep-ph/ 0201195$].$ 